\title{
Development and validation of a risk stratification model for predicting the mortality of acute kidney injury in critical care patients
}

\author{
Haofan Huang ${ }^{1 \#}$, Yong Liu ${ }^{2 \#}$, Ming $\mathrm{Wu}^{3}$, Yi Gao ${ }^{1}$, Xiaxia Yu${ }^{1}$ \\ ${ }^{1}$ School of Biomedical Engineering, Health Science Center, Shenzhen University, Shenzhen, China; ${ }^{2}$ Department of Intensive Care Unit, Shenzhen \\ Hospital, Southern Medical University, Shenzhen, China; ${ }^{3}$ Department of Critical Care Medicine \& Infection Prevention and Control, The Second \\ People's Hospital of Shenzhen \& First Affiliated Hospital of Shenzhen University, Health Science Center, Shenzhen, China \\ Contributions: (I) Conception and design: H Huang, Y Liu; (II) Administrative support: M Wu; (III) Provision of study materials or patients: Y Gao; (IV) \\ Collection and assembly of data: X Yu, M Wu; (V) Data analysis and interpretation: X Yu, H Huang; (VI) Manuscript writing: All authors; (VII) Final \\ approval of manuscript: All authors. \\ "These authors contributed equally to this work. \\ Correspondence to: Xiaxia Yu; Yi Gao. School of Biomedical Engineering, Health Science Center, Shenzhen University, Shenzhen, China. \\ Email: xiaxiayu@szu.edu.cn; gaoyi@szu.edu.cn.
}

Background: This study aimed to develop and validate a model for mortality risk stratification of intensive care unit (ICU) patients with acute kidney injury (AKI) using the machine learning technique.

Methods: Eligible data were extracted from the Medical Information Mart for Intensive Care (MIMICIII) database. Calibration, discrimination, and risk classification for mortality prediction were evaluated using conventional scoring systems and the new algorithm. A 10-fold cross-validation was performed. The predictive models were externally validated using the eICU database and also patients treated at the Second People's Hospital of Shenzhen between January 2015 to October 2018.

Results: For the new model, the areas under the receiver operating characteristic curves (AUROCs) for mortality during hospitalization and at 28 and 90 days after discharge were $0.91,0.87$, and 0.87 , respectively, which were higher than for the Simplified Acute Physiology Score (SAPS II) and Sequential Organ Failure Assessment (SOFA). For external validation, the AUROC was 0.82 for in-hospital mortality, higher than SOFA, SAPS II, and Acute Physiology and Chronic Health Evaluation (APACHE) IV in the eICU database, but for the 28- and 90-day mortality, the new model had AUROCs (0.79 and 0.80, respectively) similar to that of SAPS II in the SZ2 database. The reclassification indexes were superior for the new model compared with the conventional scoring systems.

Conclusions: The new risk stratification model shows high performance in predicting mortality in ICU patients with AKI.

Keywords: Acute kidney injury (AKI); intensive care unit (ICU); machine learning; in-hospital mortality; severity score

Submitted Aug 06, 2020. Accepted for publication Nov 26, 2020.

doi: $10.21037 / \mathrm{atm}-20-5723$

View this article at: http://dx.doi.org/10.21037/atm-20-5723 


\section{Introduction}

Acute kidney injury (AKI) is a common but complex disease in critically ill patients, leading to high morbidity and mortality $(1,2)$. In addition, AKI is associated with increased length of hospital stay (LOS), total health-related costs, and mortality (3-6). In-hospital mortality in patients with AKI has recently been estimated to be between $20 \%$ and $25 \%$ $(7,8)$, while critically ill patients with AKI requiring dialysis have a mortality rate exceeding $50 \%(9,10)$. Currently, the diagnosis criteria for AKI mostly depend on serum creatinine ( $\mathrm{SCr}$ ) assessment (3), and diagnosing AKI based on SCr might delay AKI detection, leading to a more advanced stage at diagnosis and irreversible damage and loss of organ function (11). Therefore, the development of new predictive models for risk stratification in patients with AKI in the intensive care unit (ICU) is crucial in reducing unnecessary kidney stress and improving patient outcomes $(4,12)$.

The burden of care for critically ill patients is massive. The basis of critical care is a risk stratification approach for classifying patients by severity levels and thus optimizing personal care. Precision delivery is based on this predictionpersonalization approach, deployed precisely at the right moment in the course of clinical management for improved clinical outcomes $(13,14)$. Traditionally, several rulebased severities scoring systems, and their modifications, are used based on the clinical experiences of physicians; those systems include, for example, the Sequential Organ Failure Assessment (SOFA), Simplified Acute Physiology Score (SAPS II), and Acute Physiology and Chronic Health Evaluation (APACHE) IV $(15,16)$.

Machine-learning techniques are being increasingly used to examine the risk stratification of patients $(17,18)$. Novel machine learning applications can offer improved predictive performance by maximally leveraging large-scale, complex electronic health record (EHR) and identifying the most robust signals within the noise $(19,20)$. They can rapidly assess voluminous and complex data to identify clinically relevant risk levels by applying computationally intensive statistical modeling $(19,20)$. Nevertheless, none of these models reaches sufficient precision to be used for an individual patient (21). In addition, studies have unanimously concluded that non-parametric methods might perform at least as well, if not better, than standard logistic regression-based techniques in predicting ICU mortality (22). Therefore, more robust methods for staging $\mathrm{AKI}$ are required (23).
Therefore, this study aimed to develop a risk stratification system for ICU patients with AKI at admission using machine-learning. We present the following article in accordance with the TRIPOD reporting checklist (available at http://dx.doi.org/10.21037/atm-20-5723).

\section{Methods}

\section{Study population}

This study is a cohort retrospective study based on three different population. Three databases were used: the publicly available Medical Information Mart for Intensive Care (MIMIC-III) database (24) (internal validation), the publicly available eICU Collaborative Research Database (external validation), and Shenzhen Second Renmin Hospital database (SZ2) (also external validation). In this study, all patients diagnosed with AKI in the ICU according to the diagnostic criteria described in the "Kidney Disease Improving Global Outcomes" (KDIGO) clinical practice guidelines were included according to the diagnostic codes in the hospital information system (25). Therefore, the diagnosis of AKI was: (I) increase in $\mathrm{SCr} \geq 0.3 \mathrm{mg} / \mathrm{dL}$ within $48 \mathrm{~h}$ or $\geq 50 \%$ within 7 days, and (II) urine output $<0.5 \mathrm{~mL} / \mathrm{kg} / \mathrm{h}$ for $6 \mathrm{~h}$. Baseline $\mathrm{SCr}$ was defined as the mean creatinine level before hospital admission within 3 months (25-27). If the patients had no available data, then the SCr was estimated using the back-calculation method from the Modification of Diet in Renal Disease (MRDR) formula with a GFR of $75 \mathrm{~mL} / \mathrm{min} / 1.73 \mathrm{~m}^{2}$ (28). The patients who had been hospitalized once or more were enrolled. The patients who received dialysis (renal replacement) in the ICU were identified (29) and excluded.

The predictive model was built and internally validated using data extracted from the publicly available MIMICIII database (24). During the model building process, $10 \%$ of the data from the MIMIC cohort were not used for model building but for the validation process. Hence, it was defined as an internal validation (30).

External validation of the predictive performance of the developed algorithm was evaluated using the above metrics but in a completely independent dataset. The data used for external validation were extracted from the publicly available eICU Collaborative Research Database and SZ2. The eICU is a multi-center ICU database with high granularity data for over 200,000 admissions to ICUs monitored by the eICU Programs across the United States. It was created by the Laboratory for Computational 
Physiology (LCP) at the Massachusetts Institute of Technology (MIT) and the eICU Research Institute (eRI) at Philips Healthcare (31). A total of 1,087 events made by a total of 1,063 patients were included from the eICU database.

The other external dataset was obtained from our local hospital, and a total of 84 randomly selected patients with AKI admitted to the general adult ICU in the Second People's Hospital of Shenzhen (a tertiary-care teaching hospital) from January 2015 to October 2018 were included. The study was approved by the ethics committee of the Second People's Hospital of Shenzhen (\#20180515001). For the data from this hospital, informed consent was obtained from all patients or their families by telephone. All patient privacy data were protected under the confidentiality policy.

For eICU and SZ2, the inclusion criteria were the septic shock 3.0 criteria: (I) persisting hypotension requiring vasopressors to maintain mean arterial pressure (MAP) $\geq 65 \mathrm{mmHg}$, and (II) blood lactate $>2 \mathrm{mmol} / \mathrm{L}$ despite adequate volume resuscitation. The exclusion criteria were (I) $<18$ years of age, (II) history of chronic kidney disease and kidney transplantation, (III) end-stage malignant tumors, (IV) septic shock occurred $24 \mathrm{~h}$ after admission, (V) death within $12 \mathrm{~h}$ of admission despite cardiopulmonary and brain resuscitation, (VI) missing data (without creatinine in $24 \mathrm{~h}$ after admission), and (VII) trauma and other causes of renal contusion injury. In SZ2, the patients were followed by phone calls every week.

The assessed features included baseline demographic features, vital signs, laboratory measurements, medications, and diagnostic codes. The averaged laboratory data (since a given indicator might have been measured more than once during the first $24 \mathrm{~h}$ ) included in the model were those obtained within $24 \mathrm{~h}$ of admission to the ICU. The averaged values for biochemical parameters within $24 \mathrm{~h}$ of admission to the ICU were used for downstream analysis. If variables were missing, for laboratory results, we used the mean of the normal range; for the absent height and weight, we used age and sex to stratify the liner relationship then interpolation; for the other categorical variables, we used "null" as the default value.

The study was conducted in accordance with the Declaration of Helsinki (as revised in 2013). The study was approved by the institutional ethics board of the Second People's Hospital of Shenzhen (No. 20180515001), and individual consent for this retrospective analysis was waived. The flowchart of data processing is shown in
Figure 1.

\section{Prediction algorithm}

In this study, the proposed algorithm was built based on the random forest algorithm method (32) with improvement in feature selection (Figure 2) and consisted of two steps:

(I) Feature selection. Because the relative rank of each feature could be used to reflect its relative significance (12-14), a random forest algorithm was first applied to rank the contributions of the various features. Then, a stepwise multivariable regression analysis was performed to further screen the selected variables. Variables with $\mathrm{P}<0.10$ were included in the final model building.

(II) Model building. The risk stratification model was constructed based on the selected feature subspaces using the random forest algorithm. In addition, we developed a new version of the SAPS II score by fitting a main-term logistic regression model to our data using the same explanatory variables as those used in the original SAPS II score. The same procedure was used to build a new version of the APACHE IV and SOFA scores. Mortality prediction based on the traditional scores was obtained by regressing hospital mortality on the scores using a main-term logistic regression (22). The model was written in the Python scripting language (v3.6.5) [The Python Software Foundation (PSF), https:// www.python.org; Wilmington, DE, USA].

\section{Performance evaluation}

In this study, the predictive performances of scores yielded by the current and conventional models were comparatively assessed. A 10-fold cross-validation was performed in all experiments for the different classification methods. In addition to the area under the receiver operating characteristic curve (AUROC), accuracy, specificity, sensitivity, and F-measure (F1) were also assessed to evaluate the performance of the constructed model. Discrimination was evaluated using AUROC, as well as the box plots of predicted probabilities of death for survivors and nonsurvivors, and the corresponding discrimination slopes, defined as the differences between the mean predicted risks in survivors and non-survivors. The calibration of the predictive model was assessed by the conventional Hosmer-Lemeshow test. Under perfect calibration, a 

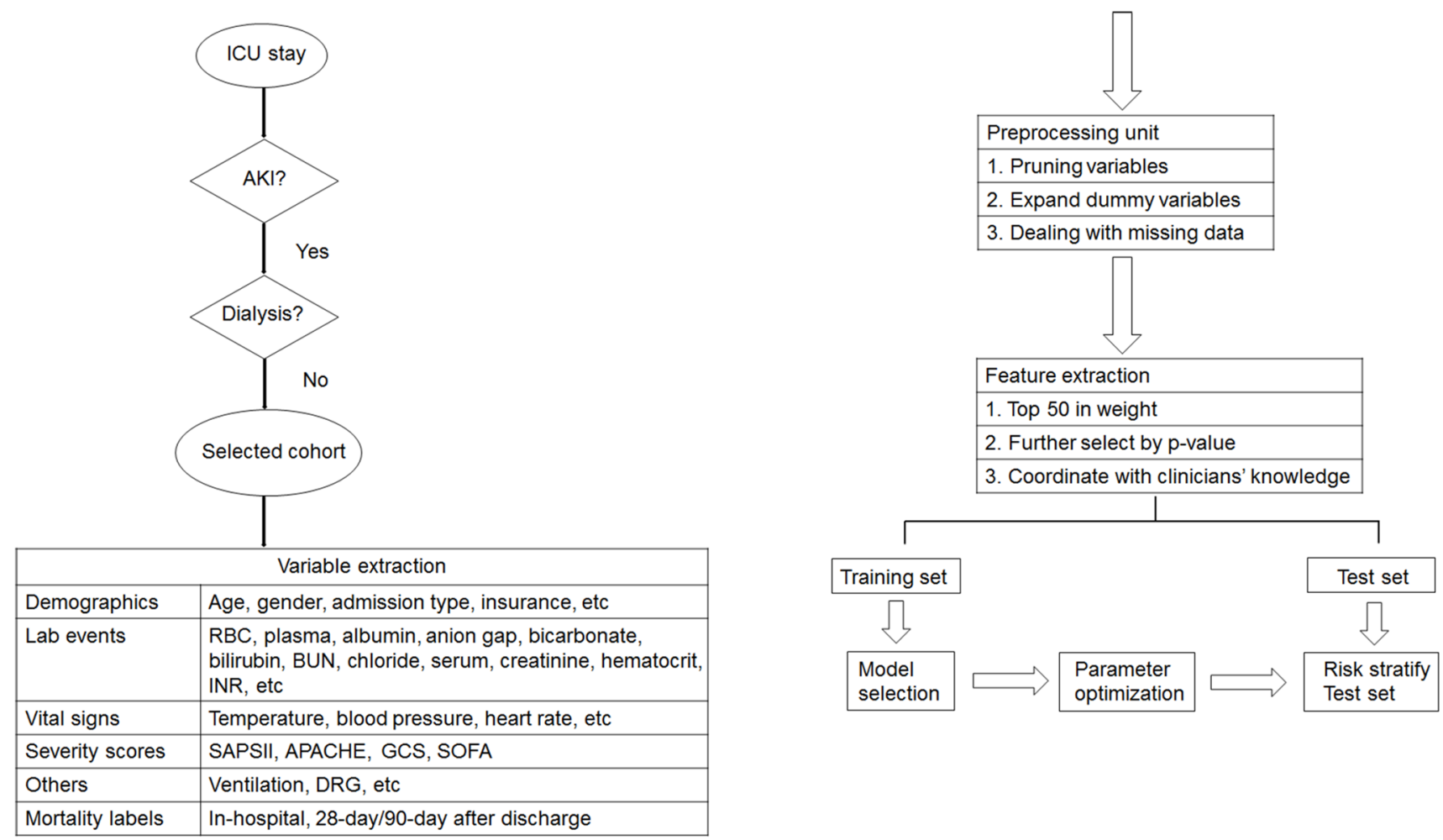

Figure 1 Flowchart of data processing. ICU, intensive care unit; AKI, acute kidney injury; RBC, red blood cell; BUN, blood urea nitrogen; INR, international normalized ratio; SOFA, Sequential Organ Failure Assessment; SAPS II, Simplified Acute Physiology Score II; APACHE, Acute Physiology and Chronic Health Evaluation.

prediction algorithm would satisfy the logistic regression equation (22). Summary reclassification measures, including continuous net reclassification index (cNRI) and integrated discrimination improvement (IDI), were relative metrics that have been designed to overcome the limitations of the usual discrimination and calibration measures (33-35). We computed the reclassification tables and associated summary measures to measure reclassification for the various methods. Finally, we used decision curve analysis (DCA) (36) to estimate the clinical usefulness and net benefit of different prediction models to facilitate the comparison among them.

\section{Statistical analysis}

Exploratory data analysis was performed for each selected variable. Median values with interquartile ranges or means with standard deviations (SDs) were calculated for continuous variables. Percentages were determined for categorical variables. Patient demographics, vital signs, laboratory results, and admission diagnoses were compared among databases by the Kruskal-Wallis rank-sum test, chisquare test, or Fisher's exact test, as appropriate. $\mathrm{P}<0.05$ was considered statistically significant. Statistical analyses were performed using Python 3.7.0 and R 3.6.3 Bell Labs (New Providence, NJ, USA) and EmpowerStats (www. empowerstats.com) on May 16, 2020.

\section{Results}

\section{Patient characteristics}

There were 3,411 patients with AKI were included from the MIMIC-III population (development set), with a total of 3,540 encounters, 1,063 patients with AKI were included from the eICU, with a total of 1,087 encounters, and 84 patients with AKI were included from the SZ2 (external validation sets). There was some heterogeneity among the different patient cohorts. All patient features are shown in Table 1. 


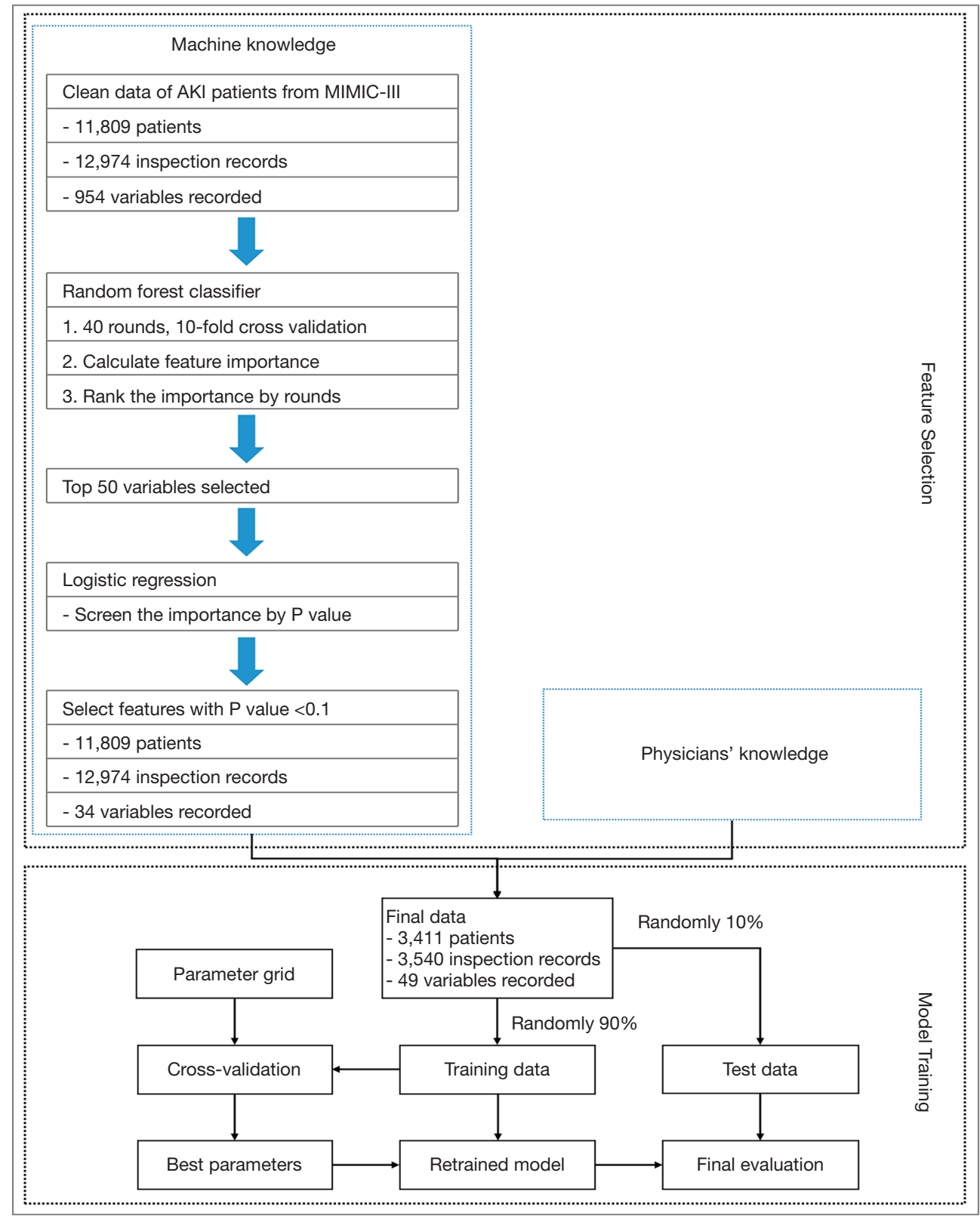

Figure 2 Building procedure for the proposed risk stratification algorithms. The following features were finally selected for model building: age, gender, length of stay in intensive care unit (ICU) (los_icu), weight, height, body mass index (BMI), diabetes, heart-related diseases, lung-related diseases, no comorbidity, has two diseases, more than three diseases, hypertension, Simplified Acute Physiology Score II (SAPS II). The follow features were collected within $24 \mathrm{~h}$ after ICU admission: albumin serum (ALB), alanine aminotransferase (ALT), aspartate aminotransferase (AST), white blood cell (WBC), platelet count (PLT), neutrophil (NEUT), lymphocyte (LY), serum total bilirubin (TBIL), serum creatinine (SCr), blood urea nitrogen (BUN), red blood cell (RBC), hemoglobin (HBG), hematocrit (HCT), activated partial thromboplastin time (PTT), prothrombin time (PT), international normalized ratio (INR), neutrophils/lymphocyte ratio (NLR), platelet/ lymphocyte ratio (PLR), $\mathrm{PCO}_{2}, \mathrm{PO}_{2}$, temperature, respirate, mean blood pressure (MEANBP), heart rate, glucose, SpO ${ }_{2}$, diastolic blood pressure (DIASBP), $\mathrm{CO}_{2}$, chloride (CL), lactate, sodium, plasma cells, mechanic ventilation (MV), min Glasgow coma scale (MINGCS), specimen. 
Table 1 Characteristics of the study population

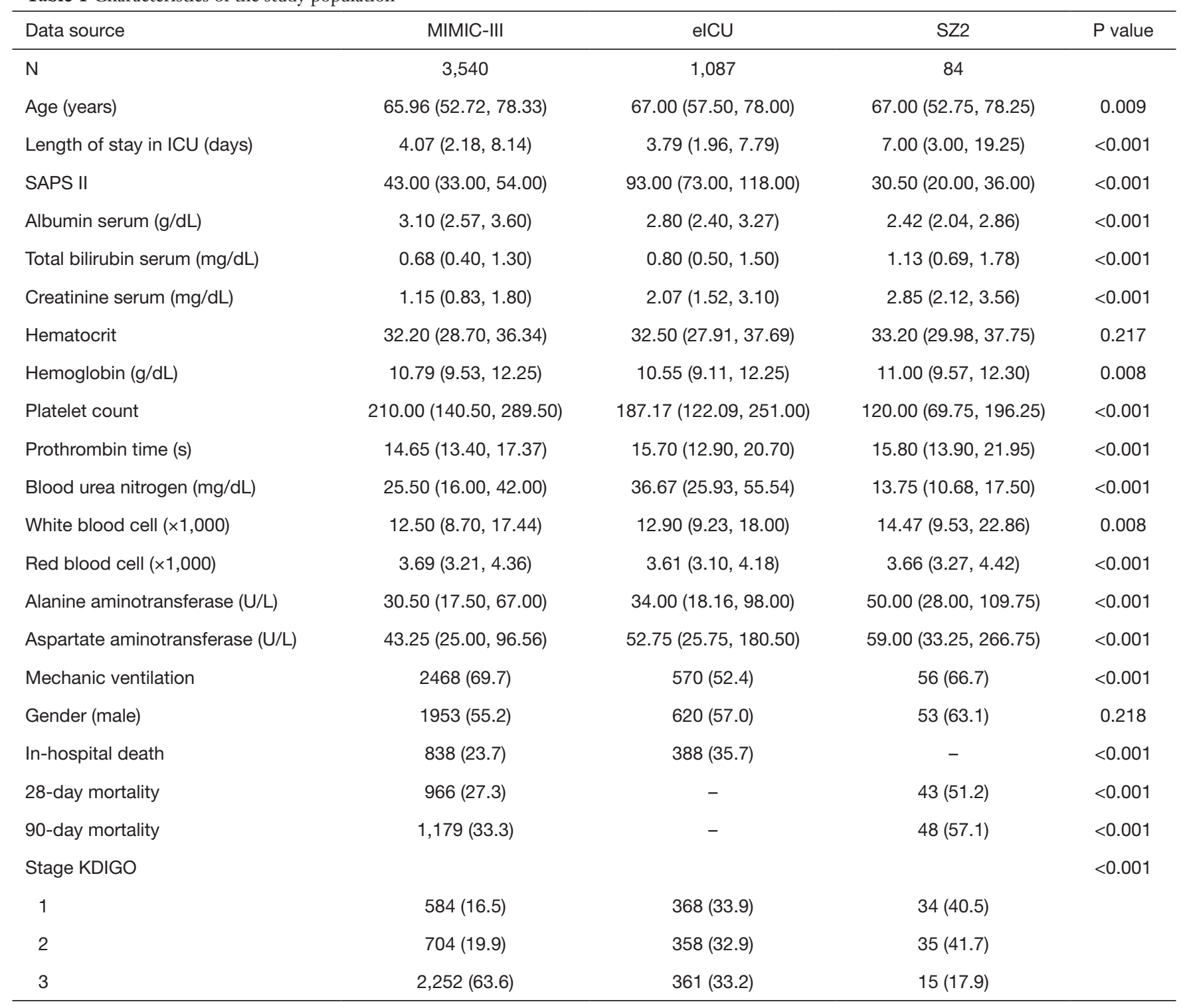

Data are median (Q1-Q3), or $\mathrm{n}(\%)$. P value: continuous variables were assessed by the Kruskal Wallis rank sum test, and count variables with a theoretical number $<10$ by the Fisher's exact probability test. ICU, intensive care unit.

\section{Feature selection}

In most cases, models built on multiple variables perform better in prediction, but it is more cost-effective and efficient to use a few but important features. Since features built on the top of trees contribute more to predicting AKI in at-risk patients, the relative importance of each feature is provided in Figure 3.

\section{Model building and validation performance}

Three predictive models were built based on the above procedure (Figure 2). Feature selection was first conducted by machine learning, then the features were further selected after discussion with our physicians, unfavorable features were deleted and useful features were added before training. These three predictive models were targeted 


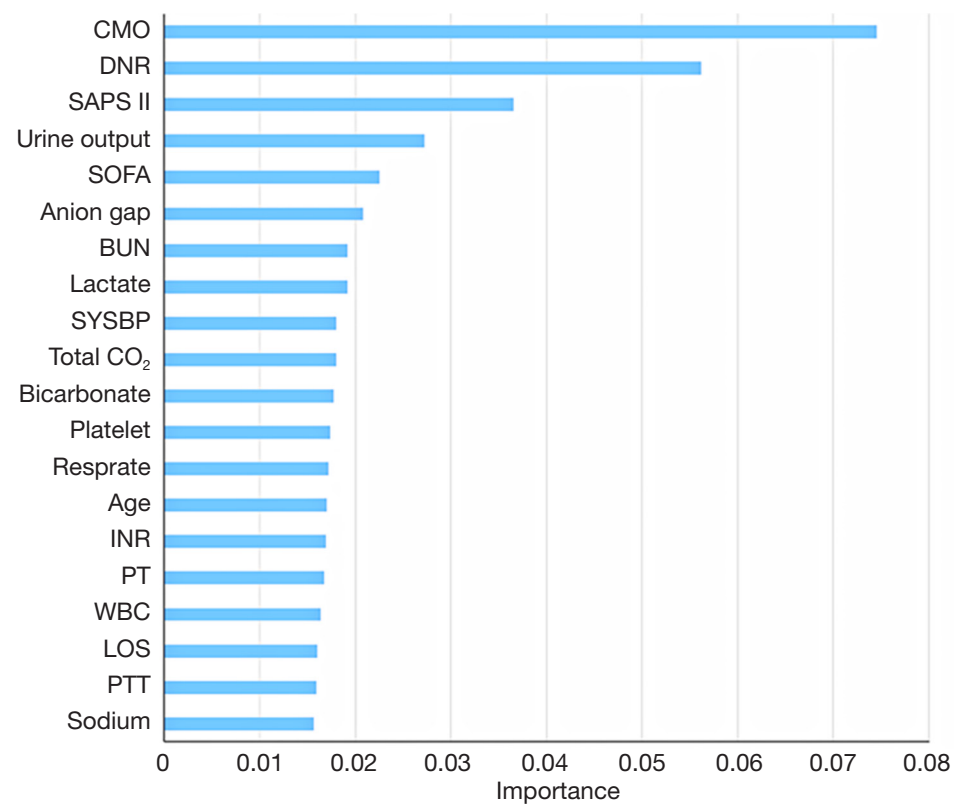

Figure 3 Variables selected by machine knowledge, ranking by their importance levels. AKI, acute kidney injuries. CMO, comfort measures only; DNR, do not resuscitate; SAPS II, Simplified Acute Physiology Score II; SOFA, Sequential Organ Failure Assessment; BUN, blood urea nitrogen; SYSBP, systolic blood pressure; INR, international normalized ratio; PT, prothrombin time; WBC, white blood cells; LOS, length of stay; PTT, partial thromboplastin time.

on identifying patient mortality during hospitalization (Model_inpatient) and at 28 (Model_28) and 90 (Model_90) days after discharge. The AUROCs for Model_inpatient, Model_28, and Model_90 were 0.91, 0.87, and 0.87, respectively. Compared with traditional scoring systems (SAPS II and SOFA), all three newly developed models had better predictive performances based on AUROCs (Figure 4A,B,C).

The performances of the three models were externally validated in the eICU and SZ2 dataset. Since eICU only has inpatient records, while SZ2 only records post-discharge mortality, Model_inpatient was evaluated in the eICU dataset and Model_28 and Model_90 in the SZ2 dataset. The ROC curves for hospital mortality prediction in the external validation are provided in Figure 4D,E,F. For the eICU dataset, AUROCs were 0.67 and 0.73 for the SOFA and SAPS II scores, respectively; similar results were obtained for the APACHE IV, which yielded an AUROC of 0.74 . Model_inpatient substantially outperformed the traditional scoring systems. The AUROC was 0.82 , revealing a clear advantage of the newly developed algorithm over traditional scores (Figure 4D). For the SZ2 dataset, the performance of Model_28 and Model_90 behaved similarly compared to SAPS II (Figure 4E,F).
The accuracies, specificities, sensitivities, and F1 scores of the new risk stratification model for internal and external validation are shown in Figure 5. Even though external validation performed well, internal validation results were slightly better in general.

\section{Discrimination and calibration}

To better evaluate the discrimination potential of our predicted results, the whole population was grouped into five categories based on predicted risk scores. The observed mortality rate was determined for each group. As demonstrated in Table 2, the lower the relative rate of patient mortality, the lower the predicted risk score, especially in patients with risk scores greater than $60 \%$ or lower than $20 \%$. These results suggested that the new algorithm could successfully identify survivors and nonsurvivors, especially among patients with very high $(0.8-1.0)$ and low $(<0.2)$ risk scores.

Discrimination was also evaluated by assessing differences between the predicted probabilities of death among survivors and non-survivors using each prediction algorithm (Figure 6). The discrimination slopes were 0.085 for the SOFA score, 0.16 for the SAPS II score, 0.257 for 
A

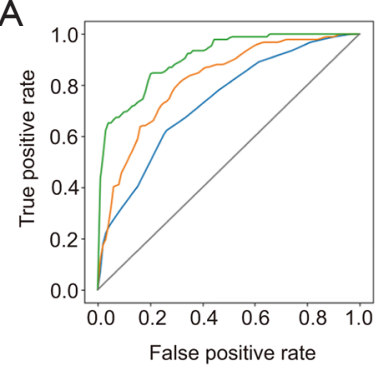

C

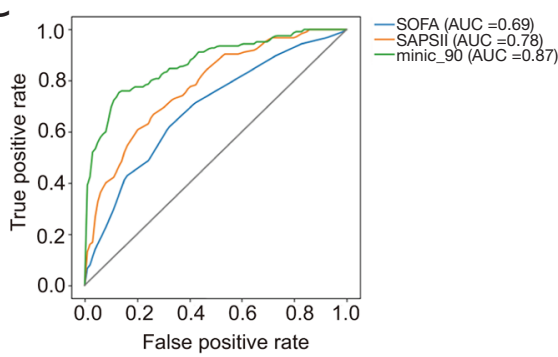

E

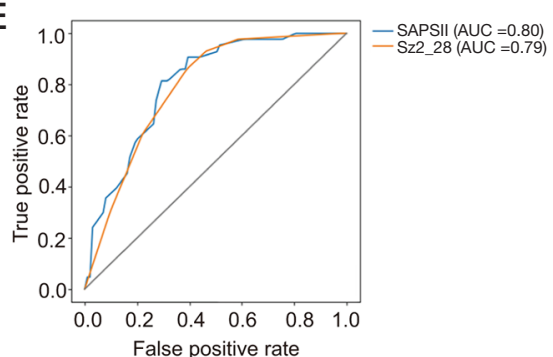

B

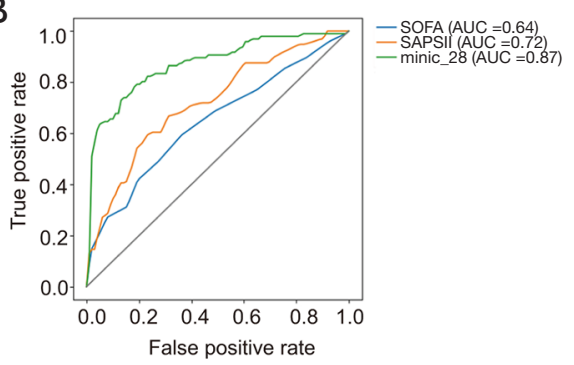

D

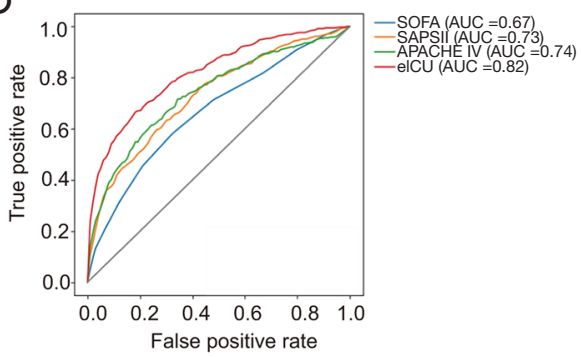

$\mathrm{F}$

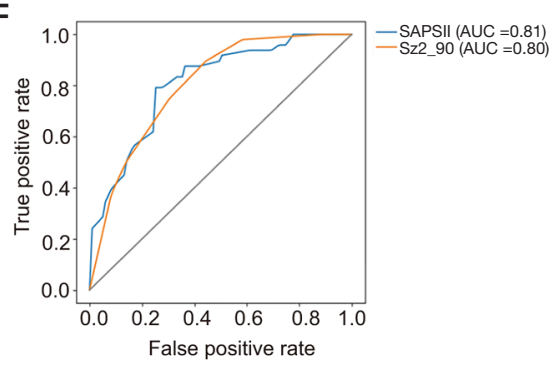

Figure 4 Performances of the newly developed prediction models and traditional scoring systems for internal and external validation. Internal validation performance: The new predictive model targeted on identifying patient mortality in the MIMIC-III database during hospitalization (A), and at 28 (B) and 90 (C) days, versus the SOFA and SAPS II models. External validation performance: (D) the new predictive model targeted on identifying patient mortality during hospitalization in eICU database, versus the SOFA, SAPS II, and APACHE IV models. (E) The new predictive model targeted on identifying 28-day mortality after discharge in the SZ2 database, versus the SAPS II model. (F) The new predictive model targeted on identifying 90-day mortality after discharge in the SZ2 database, versus the SAPS II model. SOFA, Sequential Organ Failure Assessment; SAPS II, Simplified Acute Physiology Score II; APACHE, Acute Physiology and Chronic Health Evaluation.

the APACH IV score, and 0.32 for the RF score. The plots indicated a lack of fit for the traditional scores.

Prediction based on the RF exhibited excellent calibration properties (Figure 6), as reflected by a Brier scores of $0.159\left(\chi^{2}=4.185, \mathrm{P}=0.84\right)$ for Model_inpatient, $0.177\left(\chi^{2}=0.064, \mathrm{P}=0.969\right)$ for Model_28, and 0.170 $\left(\chi^{2}=0.472, \mathrm{P}=0.79\right)$ for Model_90. The calibration plots indicated a lack of fit for the traditional scoring methods. The newly developed algorithm performed better for the entire range of death probability.

\section{Reclassification}

We calculated the risk of each individual in the entire external validation cohort and divided all patients into three groups based on the risk cut-off at $95 \%$ sensitivity and $95 \%$ specificity (37). The reclassification tables involving the RF score and traditional scores are provided in Table 3. The 

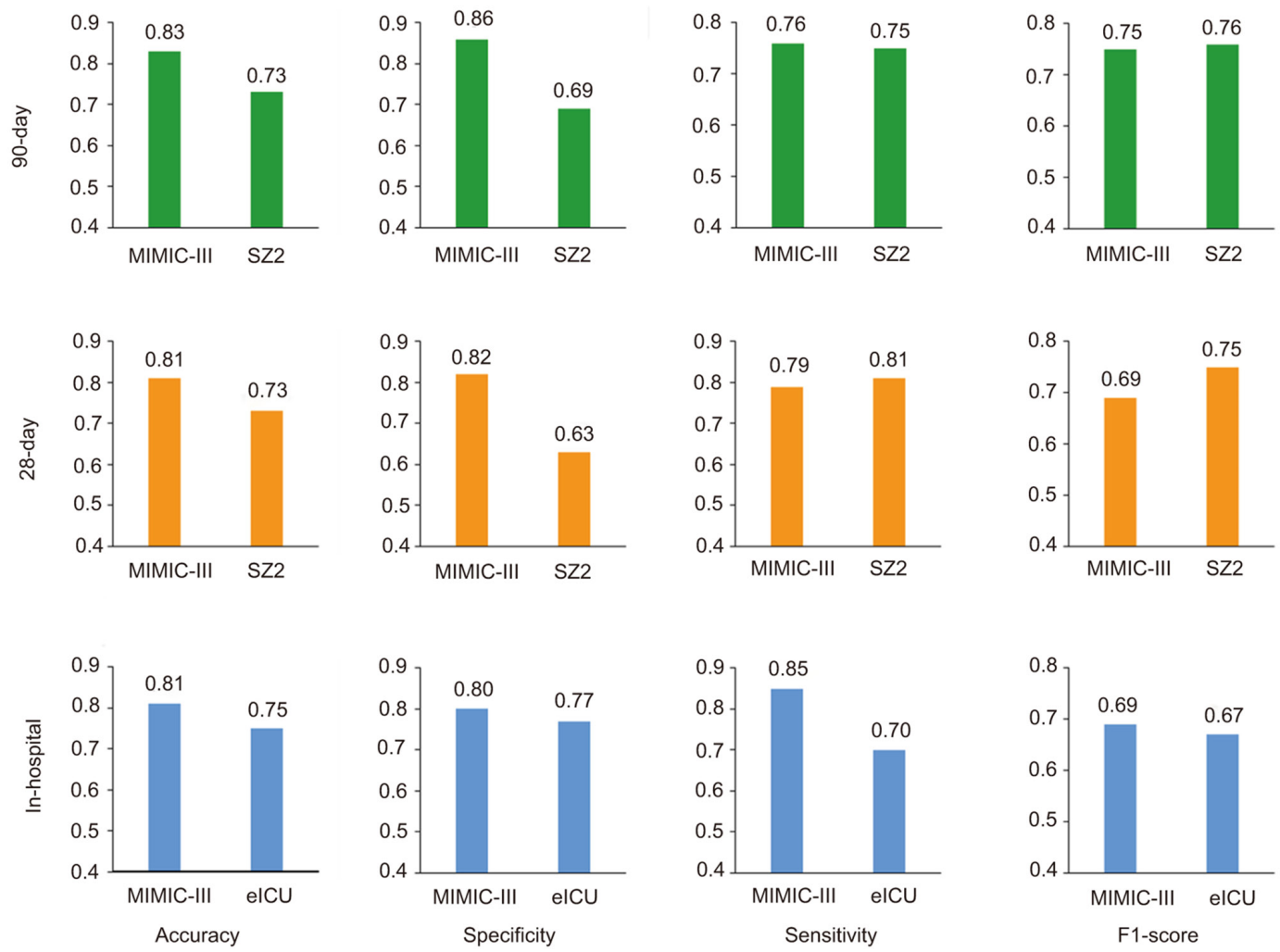

Figure 5 Predictive performances of the new proposed models. Predictive performances measured by accuracy, specificity, and sensitivity of the three predictive models for mortality during hospitalization, and at 28 and 90 days after discharge, respectively, based on the internal MIMIC-III database and two external validation datasets (the eICU database for mortality during hospitalization, and the SZ2 dataset for 28/90-day mortality).

Table 2 Relative risk ratios for various risk groups in both external validation datasets

\begin{tabular}{|c|c|c|c|c|c|c|c|c|c|}
\hline Risk & \multicolumn{3}{|c|}{ Model_inpatient } & \multicolumn{3}{|c|}{ Model_28 } & \multicolumn{3}{|c|}{ Model_90 } \\
\hline$>80 \%$ & 221 & 81.40 & 2.3 & 17 & 76.50 & 1.5 & 17 & 82.40 & 1.4 \\
\hline $60-79 \%$ & 216 & 43.50 & 1.2 & 17 & 70.60 & 1.4 & 17 & 70.60 & 1.2 \\
\hline $40-59 \%$ & 216 & 26.40 & 0.7 & 16 & 62.50 & 1.2 & 17 & 70.60 & 1.2 \\
\hline$<19 \%$ & 214 & 7.50 & 0.2 & 17 & 6.90 & 0.1 & 17 & 11.80 & 0.2 \\
\hline Total & 1,087 & 35.70 & & 84 & 51.20 & & 84 & 57.10 & \\
\hline
\end{tabular}

$\mathrm{RR}$, relative ratio.

results show that the RF score proposals resulted in a large proportion of patients being reclassified.

We computed the cNRI and IDI considering RF as the updated model, and the SOFA, SAPS II, and APACHE
IV scores as the initial models. In this case, positive values for the cNRI and IDI would indicate that RF has a better discriminative ability than the traditional methods, whereas negative values indicate the opposite. The results are 
A

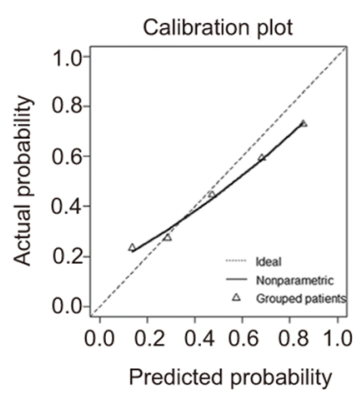

C

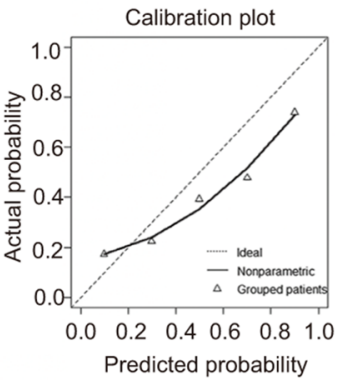

$\mathrm{E}$

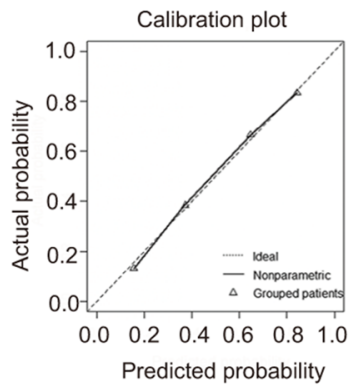

G

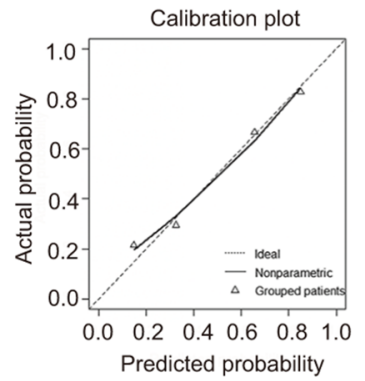

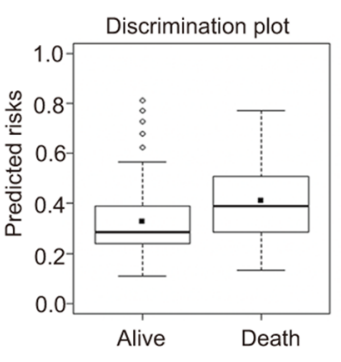
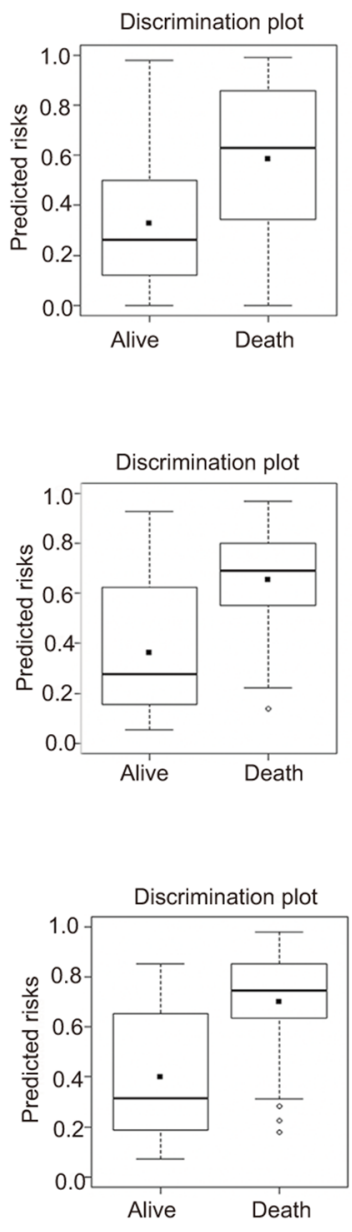

B
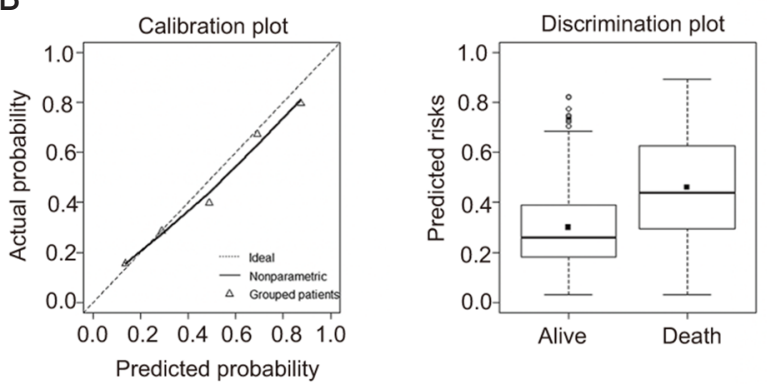

D
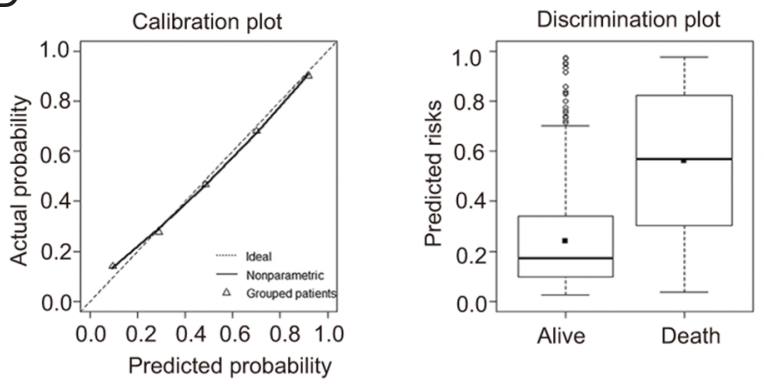

$\mathrm{F}$
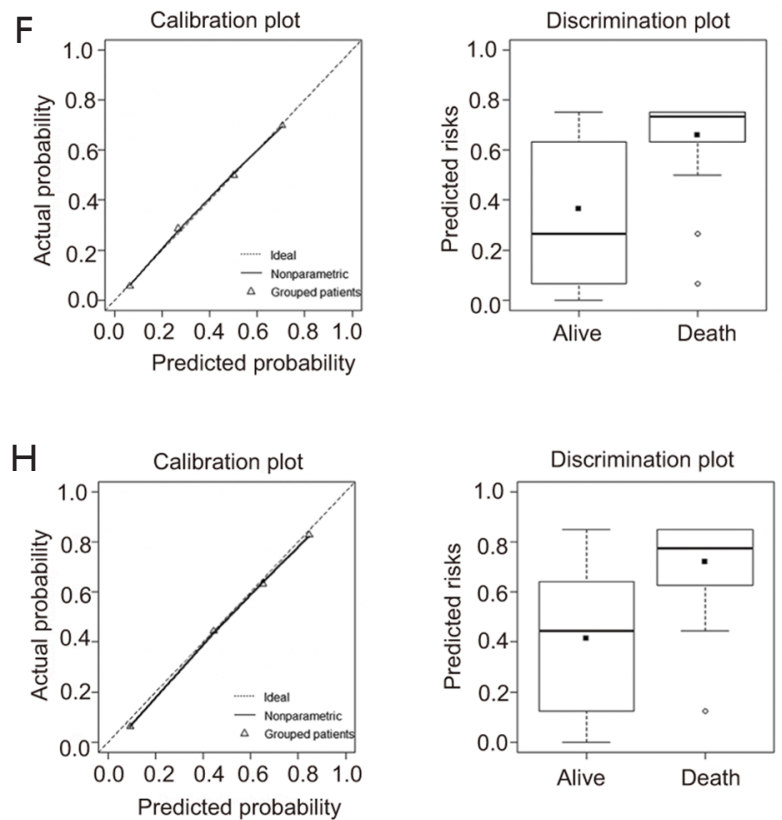

Figure 6 Calibration and discrimination potentials of the new RF-based algorithm and traditional scoring systems in external validation. (A,B,C,D) In-hospitalization mortality. (A) SOFA score (Brier score $=0.210 ; \chi^{2}=4.992, \mathrm{P}=0.759$; discrimination slope $=0.085$ ); (B) SAPS II score $\left(\right.$ Brier score $=0.192 ; \chi^{2}=7.188, \mathrm{P}=0.517$; discrimination slope $=0.16$ ); (C) APACHE IV score (Brier score $=0.203 ; \chi^{2}=249.148, \mathrm{P}=0$; discrimination slope $=0.257$ ); (D) the newly developed algorithm (Brier score $=0.159 ; \chi^{2}=4.185, P=0.84$; discrimination slope $=0.32$ ). $(\mathrm{E}, \mathrm{F})$ Mortality within 28 days after discharge. (E) 28_SAPS II score (Brier score $=0.177 ; \chi^{2}=1.181, \mathrm{P}=0.554$; discrimination slope $=0.291$ ); (F) 28_RF score (Brier score $=0.177 ; \chi^{2}=0.064, P=0.969$; discrimination slope $\left.=0.294\right)$. $(\mathrm{G}, \mathrm{H})$ Mortality within 90 days after discharge. $(\mathrm{G})$ 90_SAPS II score (Brier score $=0.172 ; \chi^{2}=0.404, \mathrm{P}=0.817$; discrimination slope $=0.302$ ); $(\mathrm{H})$ 90_RF (Brier score $=0.170 ; \chi^{2}=0.472, \mathrm{P}=0.79$; discrimination slope $=0.306$ ). SOFA, Sequential Organ Failure Assessment; SAPS II, Simplified Acute Physiology Score II; APACHE, Acute Physiology and Chronic Health Evaluation. 
Table 3 Reclassification (reclassification tables)

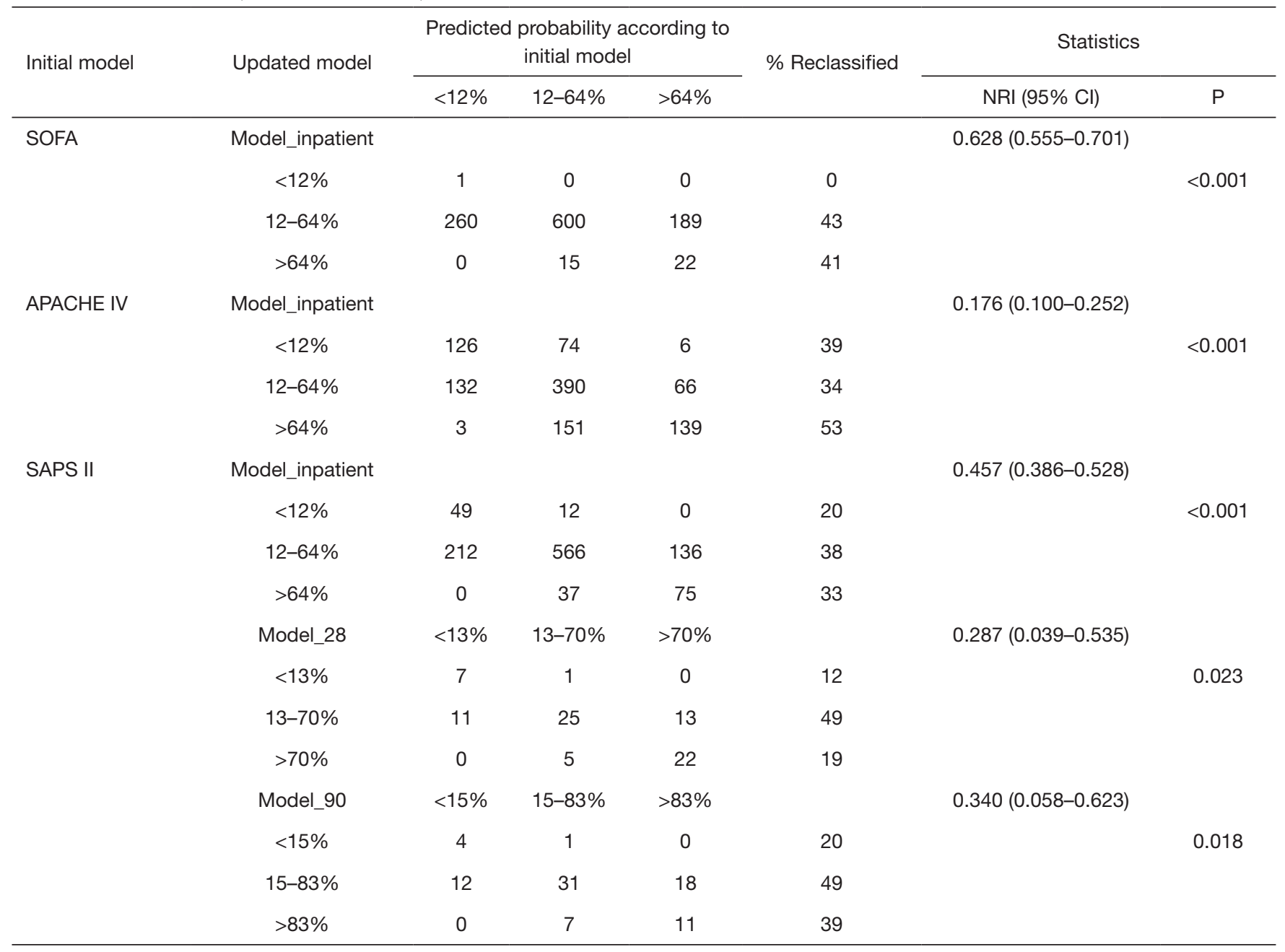

SOFA, Sequential Organ Failure Assessment; SAPS II, Simplified Acute Physiology Score II; APACHE, Acute Physiology and Chronic Health Evaluation; NRI, net reclassification index.

summarized in Table 4. Compared with the SOFA, SAPS II, and APACHE IV systems, both cNRI and IDI showed positive values for the new algorithm. These findings indicated the superiority of the newly developed model in predicting mortality in AKI patients in the ICU.

\section{Decision curve analysis}

The decision curve of the three predictive models and traditional scores is provided in Figure $7 A, B, C$. The DCA graphically shows the clinical usefulness of each model based on a continuum of potential thresholds for major risk (X-axis) and the standardized net benefit of using the model to stratify the patients (Y-axis). In this analysis, for the eICU dataset, RF provided a larger standardized net benefit across the range of major high risk compared with the SOFA, SAPS II, and APACHE IV systems (Figure 7A). For the SZ2 dataset, RF had a superior overall standardized net benefit within the wide and practical ranges of threshold probabilities, and this is similar to the performance of the SAPS II (Figure $7 B, C$ ).

\section{Discussion}

Since mortality prediction remains challenging in patients with AKI in the ICU, the current study developed and validated a machine learning technique for the risk stratification of such patients. The new risk stratification 
Table 4 Reclassification (reclassification statistics)

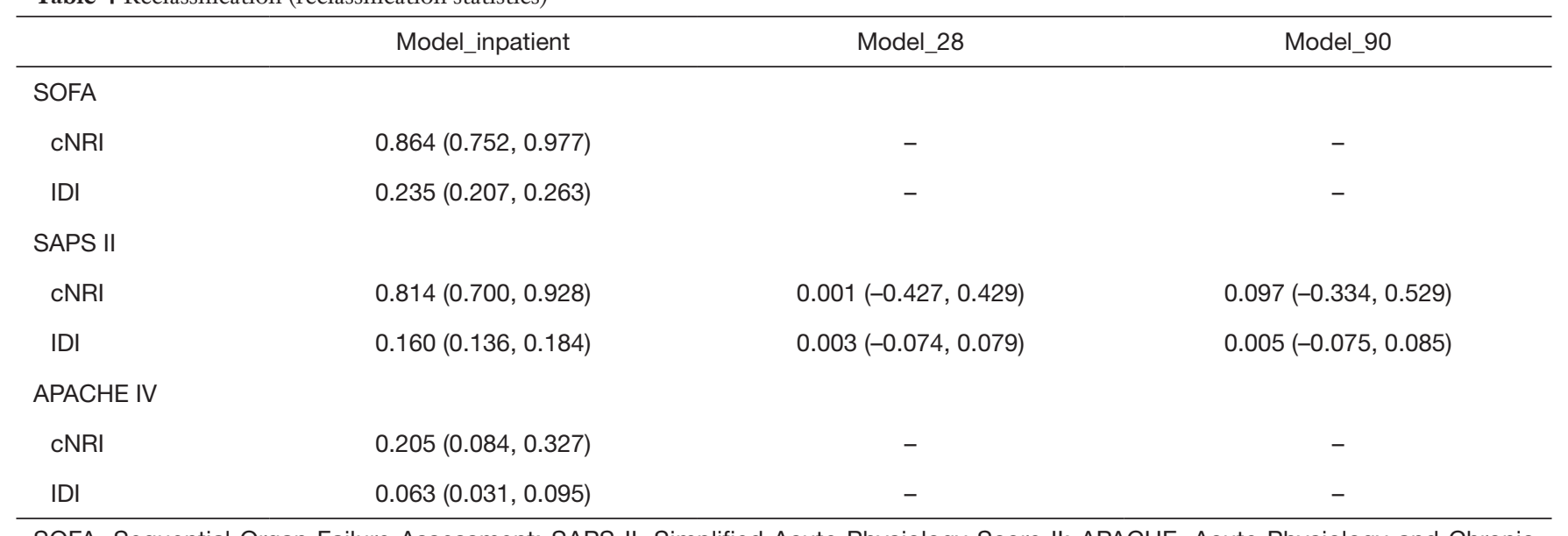

SOFA, Sequential Organ Failure Assessment; SAPS II, Simplified Acute Physiology Score II; APACHE, Acute Physiology and Chronic Health Evaluation; cNRI, continuous net reclassification index; IDI, integrated discrimination improvement.
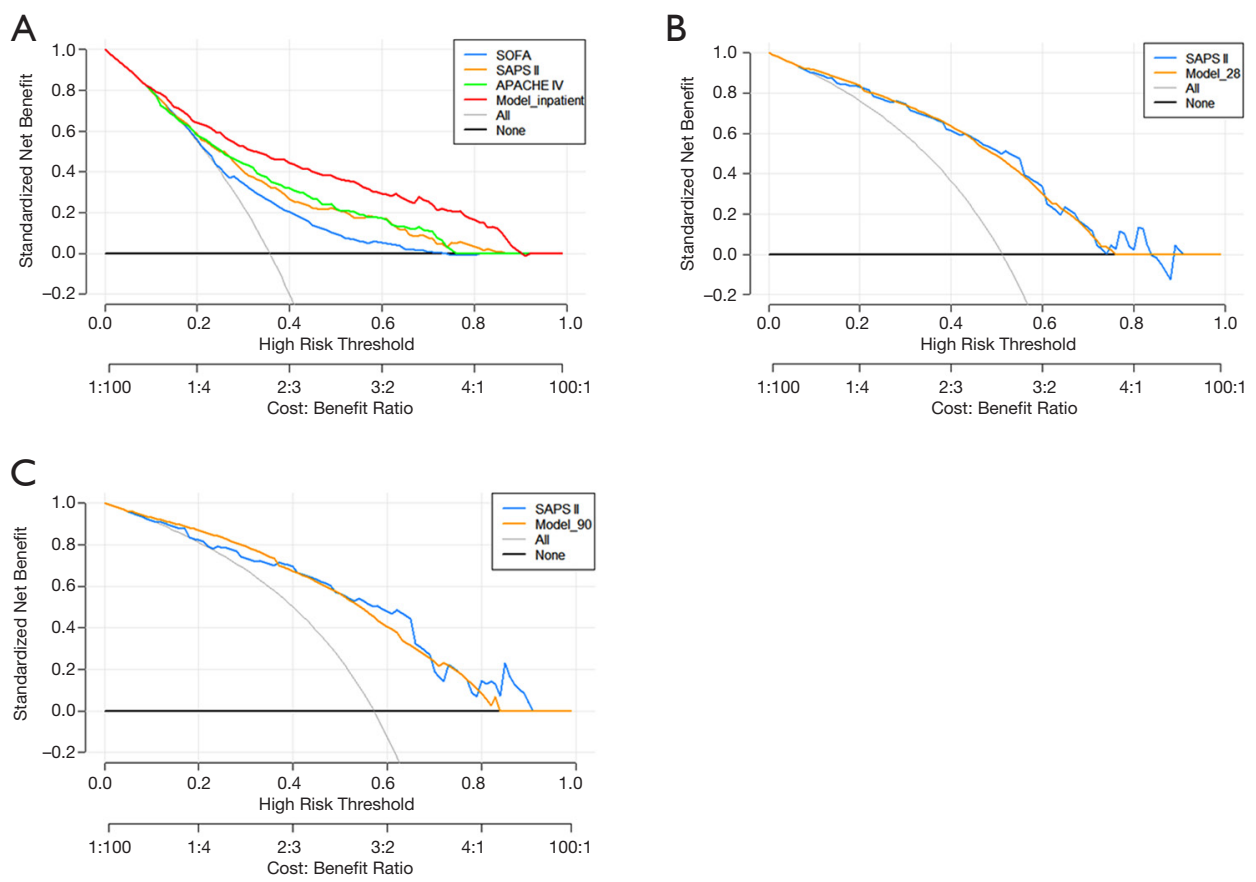

Figure 7 DCA of the new RF-based algorithm and traditional scoring systems in external validation. (A) In-hospitalization mortality; (B) mortality within 28 days after discharge; (C) mortality within 90 days after discharge. DCA, decision curve analysis.

model was superior in predicting mortality in ICU patients with AKI compared with the conventional severity scoring systems (SOFA, SAPS II, and APACHE IV) based on inhospital mortality as well as mortality at 28 and 90 days post-discharge.

Based on the retrospective MIMIC-III dataset and the
KDIGO definition of AKI (25), a tree-based predictive algorithm was proposed to build three predictive models (Model_inpatient, Model_28, and Model_90). The prediction outcomes of these three models were mortality during hospitalization and at 28 and 90 days after discharge, respectively. Their performances were validated both 
internally and externally, both in terms of discrimination and calibration, compared with traditional scoring systems. The results indicated that the tree-based predictive algorithm was robust in building prediction models for mortality during hospitalization and at 28 and 90 days after discharge. As shown above, the new model performed better than the traditional scoring systems, including APACHE IV, SOFA, and SAPS II, which are commonly used to stratify critically ill patients on the day of hospital admission $(38,39)$. The results showed that the lower the relative rate of patient mortality, the lower the predicted risk score, indicating the excellent ability of our model in grading ICU patients with AKI.

The proposed predictive model provides advantages over currently used systems. Unlike the traditional scoring systems, including SOFA, SAPS II, and APACHE IV, which assess general disease severity scores, the developed method is specific to AKI in ICU settings. Besides, the SOFA score is based on logistic regression (40). The process leading to ICU death is highly complex, and accurately predicting mortality through linear relationships with explanatory variables is challenging. The newly proposed algorithm may also provide advantages over manual AKI detection methods, which might not be implemented unless a physician already suspects AKI and are subject to potential human error.

Non-parametric techniques have been advocated for the early detection of AKI and ICU mortality prediction but may not be applicable to AKI. Mohamadlou et al. have developed a decision tree-based machine learning algorithm with a strong predictive performance compared with the SOFA score in terms of AUROC (41), but these results were challenged by Kim et al. (42), who reported no clear benefit from neural networks and support vector machines compared with APACHE-IV. Rather, in the latter study, the optimal performance was achieved with a decision tree.

Interestingly, the new algorithms were externally validated for performance based on two separate datasets. In addition, its predictive ability, as well as calibration and discrimination potential, were also tested. As shown above, the current models yielded good results in both datasets. Therefore, this new algorithm might provide an efficient real-time risk stratification tool because of its accuracy and computational speed.

Based on the above findings, the newly developed model should be applied for the risk stratification of ICU patients with AKI, which would help provide appropriate care and improve the quality of life of the patients. In addition, similar specific models could be developed for various diseases to improve risk stratification.

The limitations of this study should be mentioned. First, due to incomplete datasets, the current models could not be compared to all the widely used scoring systems for predictive performance. The APACHE IV and SOFA systems serve as references in most hospitals, and performance comparison between each of these scores and the proposed algorithm should be carried out, especially in local hospitals. Second, since eICU only includes inpatient records, while the SZ2 only contained mortality information after discharge, external validation of Model_ inpatient was only possible based on eICU, while Model_28 and Model_90 could only be tested using the SZ2. Third, there were differences in baseline features of patients among the three sources, including the training (MIMICIII) and validation (eICU and SZ2) sets, which could bias the results. Nevertheless, validation was still good in the different populations, demonstrating the generalizability of these models. Third, the data from the public databases are limited. AKI stage and the changes in kidney condition during ICU stay could not be examined in this study. Finally, the sample size was relatively small in the validation cohorts. Therefore, including additional variables and enlarging the sample size for external validation would be beneficial.

\section{Conclusions}

The prediction algorithms developed in this study have a significantly improved performance compared with currently available methods, including the SOFA, SAPS II, and APACHE IV systems, constituting promising tools for building mortality prediction models in both the clinical and research settings. This model was developed to predict the in-hospital, 28-day (after discharge), and 90-day (after discharge) death risk of ICU patients who developed AKI, thus guiding the physicians to pay more attention to high-risk patients and anticipate potential complications.

\section{Acknowledgments}

Funding: This work was supported by the National Natural Science Foundation of China (No. 61601302) and the Shenzhen peacock plan (No. KQTD2016053112051497); the Startup funding for Youth Faculty by Shenzhen 
University (No. 2018009).

\section{Footnote}

Reporting Checklist: The authors have completed the TRIPOD reporting checklist. Available at http://dx.doi. org/10.21037/atm-20-5723

Conflicts of Interest: All authors have completed the ICMJE uniform disclosure form (available at http://dx.doi. org/10.21037/atm-20-5723). The authors have no conflicts of interest to declare.

Ethical Statement: The authors are accountable for all aspects of the work in ensuring that questions related to the accuracy or integrity of any part of the work are appropriately investigated and resolved. The study was conducted in accordance with the Declaration of Helsinki (as revised in 2013). The study was approved by the institutional ethics board of the Second People's Hospital of Shenzhen (No. 20180515001), and individual consent for this retrospective analysis was waived.

Open Access Statement: This is an Open Access article distributed in accordance with the Creative Commons Attribution-NonCommercial-NoDerivs 4.0 International License (CC BY-NC-ND 4.0), which permits the noncommercial replication and distribution of the article with the strict proviso that no changes or edits are made and the original work is properly cited (including links to both the formal publication through the relevant DOI and the license). See: https://creativecommons.org/licenses/by-nc-nd/4.0/.

\section{References}

1. Hobson C, Ozrazgat-Baslanti T, Kuxhausen A, et al. Cost and mortality associated with postoperative acute kidney injury. Ann Surg 2015;261:1207-14.

2. Chawla LS, Amdur RL, Amodeo S, et al. The severity of acute kidney injury predicts progression to chronic kidney disease. Kidney Int 2011;79:1361-9.

3. Pozzoli S, Simonini M, Manunta P. Predicting acute kidney injury: current status and future challenges. J Nephrol 2018;31:209-23.

4. Lewington AJ, Sayed A. Acute kidney injury: how do we define it? Ann Clin Biochem 2010;47:4-7.

5. Palevsky PM, Liu KD, Brophy PD, et al. KDOQI US commentary on the 2012 KDIGO clinical practice guideline for acute kidney injury. Am J Kidney Dis 2013;61:649-72.

6. Silver SA, Chertow GM. The Economic Consequences of acute kidney injury. Nephron 2017;137:297-301.

7. Susantitaphong P, Cruz DN, Cerda J, et al. World incidence of AKI: a meta-analysis. Clin J Am Soc Nephrol 2013;8:1482-93.

8. Selby NM, Kolhe NV, McIntyre CW, et al. Defining the cause of death in hospitalised patients with acute kidney injury. PLoS One 2012;7:e48580.

9. Palevsky PM, Zhang JH, O'Connor TZ, et al. Intensity of renal support in critically ill patients with acute kidney injury. N Engl J Med 2008;359:7-20.

10. Uchino S, Bellomo R, Morimatsu H, et al. Continuous renal replacement therapy: a worldwide practice survey. The beginning and ending supportive therapy for the kidney (B.E.S.T. kidney) investigators. Intensive Care Med 2007;33:1563-70.

11. Bagshaw SM, George C, Dinu I, et al. A multi-centre evaluation of the RIFLE criteria for early acute kidney injury in critically ill patients. Nephrol Dial Transplant 2008;23:1203-10.

12. Lieske JC, Chawla L, Kashani K, et al. Biomarkers for acute kidney injury: where are we today? Where should we go? Clin Chem 2014;60:294-300.

13. Parikh RB, Schwartz JS, Navathe AS. Beyond genes and molecules - a precision delivery initiative for precision medicine. N Engl J Med 2017;376:1609-12.

14. Che Z, Purushotham S, Khemani R, et al. Interpretable deep models for ICU outcome prediction. AMIA Annu Symp Proc 2017;2016:371-80.

15. Le Gall JR, Loirat P, Alperovitch A, et al. A simplified acute physiology score for ICU patients. Crit Care Med 1984;12:975-7.

16. Vincent JL, Moreno R, Takala J, et al. The SOFA (SepsisRelated Organ Failure Assessment) score to describe organ dysfunction/failure. Intensive Care Med 1996;22:707-10.

17. Liu VX, Prescott HC. Precision delivery in critical care: balancing prediction and personalization. In: Vincent JL. Annual update in intensive care and emergency medicine 2019. Cham: Springer, 2019:15-27.

18. Davoodi R, Moradi MH. Mortality prediction in intensive care units (ICUs) using a deep rule-based fuzzy classifier. J Biomed Inform 2018;79:48-59.

19. Friedman J, Hastie T, Tibshirani R. The elements of statistical learning. New York: Springer series in statistics, 2001.

20. Kuhn M, Johnson K. Applied predictive modeling. New 
York: Springer, 2013.

21. Salluh JI, Soares M. ICU severity of illness scores: APACHE, SAPS and MPM. Curr Opin Crit Care 2014;20:557-65.

22. Pirracchio R, Petersen ML, Carone M, et al. Mortality prediction in intensive care units with the Super ICU Learner Algorithm (SICULA): a population-based study. Lancet Respir Med 2015;3:42-52.

23. Husain-Syed F, Ronco C. The odyssey of risk stratification in acute kidney injury. Nat Rev Nephrol 2018;14:660-2.

24. Johnson AE, Pollard TJ, Shen L, et al. MIMIC-III, a freely accessible critical care database. Sci Data 2016;3:160035.

25. Kidney Disease: Improving Global Outcomes (KDIGO) Acute Kidney Injury Work Group. KDIGO Clinical Practice Guideline for Acute Kidney Injury. Kidney Inter Suppl 2012;2:1-138.

26. Ali T, Khan I, Simpson W, et al. Incidence and outcomes in acute kidney injury: a comprehensive population-based study. J Am Soc Nephrol 2007;18:1292-8.

27. Jenq CC, Tsai MH, Tian YC, et al. RIFLE classification can predict short-term prognosis in critically ill cirrhotic patients. Intensive Care Med 2007;33:1921-30.

28. Bellomo R, Ronco C, Kellum JA, et al. Acute renal failure - definition, outcome measures, animal models, fluid therapy and information technology needs: the Second International Consensus Conference of the Acute Dialysis Quality Initiative (ADQI) Group. Crit Care 2004;8:R204-12.

29. Abhyankar S, Demner-Fushman D, Callaghan FM, et al. Combining structured and unstructured data to identify a cohort of ICU patients who received dialysis. J Am Med Inform Assoc 2014;21:801-7.

30. Alba AC, Agoritsas T, Walsh M, et al. Discrimination and calibration of clinical prediction models: users' guides to the medical literature. JAMA 2017;318:1377-84.

31. Pollard TJ, Johnson AEW, Raffa JD, et al. The eICU Collaborative Research Database, a freely available multi-center database for critical care research. Sci Data 2018;5:180178.

32. Breiman L. Bagging predictors. Machine Learning

Cite this article as: Huang H, Liu Y, Wu M, Gao Y, Yu X. Development and validation of a risk stratification model for predicting the mortality of acute kidney injury in critical care patients. Ann Transl Med 2021;9(4):323. doi: 10.21037/atm-205723
1996;24:123-40.

33. Cook NR. Use and misuse of the receiver operating characteristic curve in risk prediction. Circulation 2007;115:928-35.

34. Cook NR. Statistical evaluation of prognostic versus diagnostic models: beyond the ROC curve. Clin Chem 2008;54:17-23.

35. Pencina MJ, D'Agostino RB Sr, D'Agostino RB Jr, et al. Evaluating the added predictive ability of a new marker: from area under the ROC curve to reclassification and beyond. Stat Med 2008;27:157-72; discussion 207-12.

36. Kerr KF, Brown MD, Zhu K, et al. Assessing the clinical impact of risk prediction models with decision curves: guidance for correct interpretation and appropriate use. J Clin Oncol 2016;34:2534-40.

37. Liang W, Yao J, Chen A, et al. Early triage of critically ill COVID-19 patients using deep learning. Nat Commun 2020;11:3543.

38. Sun D, Ding H, Zhao C, et al. Value of SOFA, APACHE IV and SAPS II scoring systems in predicting short-term mortality in patients with acute myocarditis. Oncotarget 2017;8:63073-83.

39. Lee JH, Hwang SY, Kim HR, et al. Effectiveness of the sequential organ failure assessment, acute physiology and chronic health evaluation II, and simplified acute physiology score II prognostic scoring systems in paraquatpoisoned patients in the intensive care unit. Hum Exp Toxicol 2017;36:431-7.

40. Schoe A, Bakhshi-Raiez F, de Keizer N, et al. Mortality prediction by SOFA score in ICU-patients after cardiac surgery; comparison with traditional prognostic-models. BMC Anesthesiol 2020;20:65.

41. Mohamadlou H, Lynn-Palevsky A, Barton C, et al. Prediction of acute kidney injury with a machine learning algorithm using electronic health record data. Can J Kidney Health Dis 2018;5:2054358118776326.

42. Kim SY, Kim S, Cho J, et al. A deep learning model for real-time mortality prediction in critically ill children. Crit Care 2019;23:279. 